\title{
Dural Sinus Thrombosis after Resection of Vestibular Schwannoma using Suboccipital Retrosigmoid Approach - Thrombosis Classification and Management Proposal.
}

Kamil Krystkiewicz ( $\nabla$ kamil.krystkiewicz@gmail.com )

10th Military Research Hospital in Bydgoszcz https://orcid.org/0000-0002-6784-1969

Dawid Wrona

Copernicus Memorial Hospital

Marcin Tosik

Copernicus Memorial Hospital

Marcin Birski

10th Military Research Hospital

Łukasz Szylberg

Nicolaus Copernicus University in Torun

Anna Morawska

10th Military Research Hospital

Jacek Furtak

10th Military Research Hospital,

Cezary Wałęsa

10th Military Research Hospital,

Konrad Stopa

10th Military Research Hospital,

Marek Harat

10th Military Research Hospital,

\section{Research Article}

Keywords: dural sinus thrombosis, vestibular schwannoma, suboccipital retrosigmoid approach, posterior fossa

Posted Date: October 27th, 2021

DOI: https://doi.org/10.21203/rs.3.rs-1019003/v1 
License: (c) (i) This work is licensed under a Creative Commons Attribution 4.0 International License. Read Full License 


\section{Abstract}

Objective

Dural sinus thrombosis is one of the complications after posterior fossa surgery. However, that topic is not described well with regard to vestibular schwannoma surgery using the unique suboccipital retrosigmoid approach.

Methods

We analyzed retrospectively medical records and radiological investigations of 116 patients. The including criteria were: histopathologically confirmed vestibular schwannoma operated on using the retrosigmoid approach, preoperative and postoperative contrast enhanced MRI, at least 1-year follow-up.

Results

The patient group included $36 \%$ males, $64 \%$ females. The average age was $47.3 \pm 13.9$ years. $60 \%$ of tumors were classified as T4b according to the Hannover scale and their mean volume was $13.73 \pm 10.28$ $\mathrm{cm}^{3}$. There were no signs of thrombosis preoperatively. In $26(22 \%)$ cases, postoperative changes in the dural sinuses were found. In $7(27 \%)$ cases there was an external compression by the hemostatic agent, in $19(73 \%)$ cases a thrombus was visualized in the sinus lumen. The size of the sinus, age, tumor size were not risk factors for thrombosis, whereas an intraoperative sinus injury was a statistically significant risk factor $(p=0.0012)$. All of the patients diagnosed with thrombosis were in good clinical condition in distant follow-up (mRankin=0). Complete recanalization was observed in $58 \%$ of cases after 1 -year follow-up.

Conclusions

Postoperative changes in the dural venous sinuses are a frequent finding after vestibular schwannoma surgery using the suboccipital retrosigmoid approach. Intraoperative dural injury is a risk factor for thrombosis. Thrombosis in that group of patients is usually asymptomatic and does not influence the prognosis.

\section{Introduction}

Dural sinus thrombosis (DST) it is a complication after posterior fossa surgery, especially in the cases of vestibular schwannoma surgery and is reported in 4.7-38.9\% $[1-3,5]$. Various intraoperative risk factors, which could cause such complication have been described: injury of the dural sinus, long duration of the surgery, surgical mobilization of the sinus, bone skeletonization around the sinus, thermal injury caused by coagulation, or even heat generated by a microscope lamp [1]. Depending on the size of the affected sinus, extent of the thrombus and individual vascular anatomy, there could be a wide range of symptoms, from clinically asymptomatic through mild, like headache to even severe, like intracerebral or intracerebellar hemorrhage, hydrocephalus and death [9]. There is no evidence-based data supporting the 
management of postoperative sinus thrombosis. The first choice approach to spontaneous cerebral sinus thrombosis is aggressive anticoagulation, however, such treatment is contraindicated in the early postoperative period, because of the risk of hematoma. This is why the treatment strategies vary from more conservative approach to administration of anticoagulants. The literature lacks information focusing on presentation of the thrombus presents in postoperative imaging, radiological classification, and there is no algorithm proposed for therapeutic approach to postoperative lateral sinus thrombosis after vestibular schwannoma resection using the suboccipital retrosigmoid approach.

\section{Materials And Methods}

\section{Data source and study design}

We retrospectively analyzed all patients operated on for cerebellopontine angle occupying lesions using suboccipital restrosigmoid approach in the single institution between January 2014 and June 2020. The inclusion criteria were as follows: 1) histopathological confirmation of the vestibular nerve schwannoma, 2) patients $\geq 18$ years old, 3 ) exclusively retrosigmoid suboccipital craniotomy or craniectomy, 4) preoperative and postoperative contrast enhanced MRI imaging available, 5) at least one follow-up imaging. We excluded the reoperations from the analysis.

A medical database was searched using the D33.3 code from the International Statistical Classification of Diseases and Related Health Problems ICD-10. Patients with a lesion occupying the cerebellopontine angle and histopathological confirmation of vestibular schwannoma were included into the study. Age, sex, comorbidities with special attention to thrombophilia and thrombosis history, size and volume of the tumor, postoperative anticoagulation therapy, symptoms of intracranial hypertension (headache, vomiting, disorders of consciousness), intraoperative injury of the dural sinus and its surgical management, time of surgery, length of hospitalization were taken into analysis. Sizes and volumes were measured using the commercial neuronavigation software (Cranial v 4.0, BrainLab, Germany).

In our institution we perform gadolinium-enhanced MRI imaging using T1-weighted sequence without and with contrast, T2 sequence, FLAIR preoperatively and postoperatively in 1 to 3 days after the surgery. In order to assess the patency of venous sinuses, contrast enhanced T1 was used. Magnetic resonance venogram was not routinely used in most cases. Radiological investigations were independently analyzed by the first author (KK) and the consulting neuroradiologists (KS, CW) and focused on the dural venous sinus postoperative changes, preoperative and postoperative tumor characteristics (size according to the Hannover grading system, preoperative and postoperative cerebellar or brainstem edema, cyst presence, volume, extent of resection), signs of intracerebellar hemorrhage and dural sinuses dominance. When a diagnosis of dural venous thrombosis was established, the location of the affected sinus, its dominance, presence of marginal flow, dilatation of the cerebellar veins were analyzed using the radiological scale prepared by the main author (KK).

For patients with radiologically diagnosed postoperative changes (compression or thrombosis) further details about the clinical course were sought. Sign consents were obtained from each study participant 
before further proceedings. The patients were examined neurologically in the outpatient clinic. Long postoperative course was investigated for early and late complications like headaches, signs of intracranial hypertension, liquorrhea or wound dehiscence. Radiological follow-up was analyzed and particular attention was paid to the width of the affected sinus, thrombus resorption and the degree of resorption.

\section{Statistical analysis}

Univariate analyses were performed to compare patient characteristics and the rate of complications between patients with and without postoperatively compromised sinus. Pearson's chi-square test and Fisher's exact test were used for analysis of categorical

data, in association with the occurrence of postoperative sinus thrombosis. Statistical significance was defined as a p-value of 0.05 or less.

\section{Ethics}

The Institutional Ethics Committee was informed about the ongoing study, and the Committee stated no need for its approval. Informed consent was obtained from all individual participants included in the study.

\section{Results}

Patients and tumor characteristics

During the analyzed period of time, 116 patients met the inclusion criteria and were included in the analysis. Among these patients, $74(64 \%)$ were women and 42 (36\%) were men. One patient had known thrombophilic coagulopathy - antiphospholipid syndrome. The mean age was $47.3 \pm 13.9$ years. The average tumor size was: $31.4 \pm 11.4 \mathrm{~mm}$ in the anteroposterior dimension, $28.8 \pm 9.8 \mathrm{~mm}$ in the mediolateral dimension, $28.6 \pm 9.9 \mathrm{~mm}$ in the superoinferior dimension. 63 tumors (54\%) were operated on the right side and $53(46 \%)$ on the left side. The mean tumor volume was $13.73 \pm 10.28 \mathrm{~cm}^{3}$. According to the Hannover scale, 4 tumors (3\%) were classified as grade T1, 4 (3\%) as grade T2, 8 (7\%) as grade T3a, 8 (7\%) as grade T3b, $23(20 \%)$ as grade T4a and 69 as grade T4b (60\%). The mean duration of the surgery was 4 hours and 44 minutes \pm 1 hour and 37 minutes. The mean length of stay (LOS) was 13,9 $\pm 9,8$ days (range 6 to 76 days).

Dural sinus thrombosis

In $26(22 \%)$ cases, changes in the contrast filling of the lateral sinuses were noted. In $7(27 \%)$ cases, the sinus was narrowed because of external compression, caused by the hemostatic agent used for the dural closure purposes (Figure 1.). It could be easily recognized when preoperative and postoperative images were compared. In the postoperative view, the internal margin of the sinus was not smooth, however, the 
sinus lumen was filled with contrasted blood. The remaining cases presented true thrombosis, with the area inside the lumen lacking the contrast filling. In 8 (31\%) cases, the thrombus filled less than a half (Figure 2.) and in 6 cases (23\%) more than a half of the sinus cross-section (Figure 3.). In 2 cases (8\%), there was almost complete closure of flow through the sinus except for narrow marginal contrast filling (Figure 4.). In 2 cases (8\%) a complete obstruction of contrast filling was observed, however, without engorgement of the draining veins (Figure 5.), which was seen in one case (3\%) (Figure 6.). Some pattern could be noted. This is why we developed a classification of postoperative changes seen in the lateral sinuses (Table 1.).

Table 1

Classification of postoperative changes in the lateral dural sinuses.

\begin{tabular}{|c|c|c|c|c|}
\hline Grade & Description & Thrombosis & $\begin{array}{l}\text { Venous } \\
\text { engorgement }\end{array}$ & Frequency \\
\hline I & $\begin{array}{l}\text { External compression, no intraluminal } \\
\text { thrombus }\end{array}$ & - & - & $\begin{array}{l}7 \text { cases } \\
(27 \%)\end{array}$ \\
\hline II & $\begin{array}{l}\text { Thrombus fills less than the } 50 \% \text { of the cross- } \\
\text { section of sinus }\end{array}$ & + & - & $\begin{array}{l}8 \text { cases } \\
(31 \%)\end{array}$ \\
\hline III & $\begin{array}{l}\text { Thrombus fills more than the } 50 \% \text { of the } \\
\text { cross-section of sinus }\end{array}$ & + & - & $\begin{array}{l}6 \text { cases } \\
(23 \%)\end{array}$ \\
\hline IV & $\begin{array}{l}\text { Almost complete closure of the sinus with the } \\
\text { marginal flow around the thrombus }\end{array}$ & + & - & $\begin{array}{l}2 \text { cases } \\
(8 \%)\end{array}$ \\
\hline V & Complete closure of the sinus & + & - & $\begin{array}{l}2 \text { cases } \\
(8 \%)\end{array}$ \\
\hline VI & $\begin{array}{l}\text { Complete closure of the sinus with } \\
\text { engorgement of draining veins }\end{array}$ & + & + & $\begin{array}{l}1 \text { case } \\
(4 \%)\end{array}$ \\
\hline
\end{tabular}

None of the patients had known thrombophilia. All cases were ipsilateral to the performed surgery. In 10 cases (38\%), the affected sinus was dominating, in 6 cases symmetric (23\%). In 12 cases (46\%) the tumor was located on the right side, in 14 on the left (54\%). Dominance of the affected sinus was not a significant factor for thrombosis $\left(\mathbb{Q}^{2}, \mathrm{p}=0.16\right)$. Among all patients, 18 were female $(69 \%)$ and 8 were male $(31 \%)$. Differences between sexes did not reach significance $\left(\mathbb{\nabla}^{2}, p=0.17\right)$. 2 patients $(8 \%)$ had T3a grade tumors, 1 patient (4\%) had a T3b grade tumor, 6 patients had T4a (23\%) and $17(65 \%)$ had T4b tumors. The tumor size in the Hannover scale was not a significant risk factor for thrombosis $\left(\mathbb{\bigotimes}^{2}, p=0.82\right)$, nor was the tumor volume $\left(13.51 \pm 11.21 \mathrm{~cm}^{3}\right.$ for the affected group and $13.6 \pm 10.1 \mathrm{~cm}^{3}, \mathrm{U}$ Mann-Whitney test, $p=0.57$ ). The mean age of patients in the thrombotic group was $46 \pm 14$ years and did not differ significantly from the non-affected group ( $U$ Mann-Whitney test, $p=0.61$ ). Mean time of surgery was also similar in both groups (U Mann-Whitney test, $\mathrm{p}=0.58$ ). Mean LOS was $14 \pm 13$ days and was similar to the control group (U Mann-Whitney test, $p=0.53$ ). The dural sinus was injured intraoperatively in 6 cases $(23 \%)$ in the thrombotic group and in $3(3 \%)$ cases in the non-affected group, and was a statistically 
significant risk factor for lateral sinus thrombosis $\left(\mathbb{\Xi}^{2}, p=0.0012\right)$. The differences between the thrombotic and non-thrombotic group are presented in Table 2.

Table 2

Analysis of the various factors in the thrombotic group.

\begin{tabular}{|c|c|c|c|}
\hline Parameter & Thrombus - 26 cases & $\begin{array}{l}\text { No thrombus - } 97 \\
\text { cases }\end{array}$ & Statistical analysis \\
\hline \multirow{3}{*}{$\begin{array}{l}\text { Sinus } \\
\text { dominance }\end{array}$} & Dominant - $10(38 \%)$ & Dominant - 46 (49\%) & \multirow[t]{3}{*}{$\square^{2}, p=0.16$} \\
\hline & Symmetric - 6 (23\%) & Symmetric - 19 (20\%) & \\
\hline & $\begin{array}{l}\text { Non-dominant - } 10 \\
(38 \%)\end{array}$ & $\begin{array}{l}\text { Non-dominant - } 28 \\
(30 \%)\end{array}$ & \\
\hline \multirow[t]{2}{*}{ Sex } & Male - $8(31 \%)$ & Male - $41(44 \%)$ & \multirow[t]{2}{*}{$\square^{2}, p=0.17$} \\
\hline & Female - 18 (69\%) & Female - $56(60 \%)$ & \\
\hline \multirow[t]{6}{*}{ Hannover scale } & T3a - $2(8 \%)$ & $\mathrm{T} 1-2(2 \%)$ & \multirow[t]{6}{*}{$\square^{2}, p=0.82$} \\
\hline & T3b - $1(4 \%)$ & T2 - $2(2 \%)$ & \\
\hline & $\mathrm{T} 4 \mathrm{a}-6(23 \%)$ & T3a - $3(3 \%)$ & \\
\hline & $\mathrm{T} 4 \mathrm{~b}-17(65 \%)$ & $\mathrm{T} 3 \mathrm{~b}-4(4 \%)$ & \\
\hline & & T4a - $13(14 \%)$ & \\
\hline & & T4b - $69(75 \%)$ & \\
\hline \multirow[t]{2}{*}{ Sinus injury } & Yes - $8(31 \%)$ & Yes - $3(3 \%)$ & \multirow[t]{2}{*}{$\square^{2}, p=0.0012$} \\
\hline & No - $16(69 \%)$ & No - 90 (97\%) & \\
\hline Age & $46 \pm 14$ years & $47 \pm 13$ years & $\begin{array}{l}\text { U Mann-Whitney test, } \\
\mathrm{p}=0.61\end{array}$ \\
\hline Tumor volume & $13,51 \pm 11,21 \mathrm{~cm}^{3}$ & $13,6 \pm 11,12 \mathrm{~cm}^{3}$ & $\begin{array}{l}\text { U Mann-Whitney test, } \\
p=0.57\end{array}$ \\
\hline LOS & $14 \pm 13$ days & $14 \pm 8$ days & $\begin{array}{l}\text { U Mann-Whitney test, } \\
p=0.53\end{array}$ \\
\hline $\begin{array}{l}\text { Length of } \\
\text { surgery }\end{array}$ & $4 \mathrm{~h} 26 \min \pm 1 \mathrm{~h} 14 \mathrm{~min}$ & $4 \mathrm{~h} 48 \mathrm{~min} \pm 1 \mathrm{~h} 11 \mathrm{~min}$ & $\begin{array}{l}\text { U Mann-Whitney test, } \\
\mathrm{p}=0.58\end{array}$ \\
\hline
\end{tabular}

None of the patients presented with the symptoms suggesting thrombosis, except one patient, who developed signs of increased intracranial pressure on the first postoperative day. However, there was a major intraoperative venous bleeding from the incidentally avulsed superior petrosal vein and thereafter from the superior petrosal sinus. In the early postoperative course, the patient developed cerebellar edema, intracerebellar hemorrhage and obstructive hydrocephalus. He was reoperated emergently, the posterior fossa decompression and external ventricular catheter were employed. After 14 days of 
treatment in the ICU, the patient died. The postoperative CT revealed massive cerebellar edema, a small intracerebellar hematoma and signs of the lateral sinus thrombosis (grade II), which was confirmed by contrast enhanced MRI. The most possible explanation was a venous stroke, caused by intraoperative, incidental closure of the superior petrosal vein during the surgery, or lateral dural sinus thrombosis. The remaining patients were classified as 5 in the GOS (19 cases, 73\%) and 4 ( 1 case, $4 \%$ ). 5 patients were lost during the follow-up (19\%). The mean follow-up time was $21,75 \pm 11,77$ months.

Postoperative anticoagulation therapy was instituted in 10 cases (38\%). In all of the treated cases, prophylactic dosage of enoxaparin was administered $24-48 \mathrm{~h}$ after the surgery and was maintained until the discharge. No complications of anticoagulation were observed.

In 7 cases (27\%), the thrombus was located only in the sigmoid sinus, in 6 cases (23\%) - exclusively in the transverse sinus, in 4 cases (15\%) - in the transverse-sigmoid junction and sigmoid sinus, in 3 cases $(12 \%)$ - in the transverse-sigmoid junction and transverse sinus, in 6 cases $(23 \%)$ - in the sigmoid, transverse and sinus junction. In 2 cases (8\%), the thrombus was located also in the dural sinuses within the tentorium.

19 patients (73\%) reached at least 12 months of the follow-up and were under long-term postoperative control. In all cases, some degree of thrombus resorption was observed. In 8 cases (42\%), there was a partial recanalization, in 11 cases (58\%), the postoperative changes resolved completely. None of them presented symptoms which could be connected with thrombosis.

\section{Discussion}

Suboccopital retrosigmoid craniotomy is an accepted approach for the vestibular schwannoma surgery. It provides wide exposure of the cerebellopontine angle and gives an opportunity to resect large tumors and preserve hearing in some cases. Lateral sinus thrombosis is a known complication after the posterior fossa surgery and has been noted in $4.7-11.6 \%$ of cases [2-8]. However, there is limited data about that complication exclusively in the vestibular schwannoma surgery. There is a series of studies $[1,4,10]$, however, they have some limitations. In most of them non-contrast CT was used as a postoperative control [1], the analyzed cohort included various surgical approaches $[1,3,10]$ or translabyrinthine approach [5]. There are no large cohort studies with well-documented postoperative radiological investigation on the retrosigmoid approach in the vestibular schwannoma surgery.

The risk factors of deep venous thrombosis after skull base surgery are well described [4]. The main concern it to prevent pulmonary embolism, which could be fatal. On the other hand, in thrombosis of the dural sinuses hyperemia and venous hypertension, which could cause intracranial hypertension and cerebral hemorrhage, are more problematic. What is more, the risk factors and management of the lateral dural venous sinuses are less evident than in the case of deep venous thrombosis.

Symptomatology 
Various symptomatology can be presented in the venous sinus thrombosis: headaches, seizures, intracranial hypertension, intraparenchymal hemorrhage $[1,6,8]$. Some cases of fatal course of sinus thrombosis have even been presented [9]. However, most studies emphasize mild or even asymptomatic course of lateral sinus thrombosis $[1,2,3,5,8]$. In our study, most of the patients, except one, were completely asymptomatic. In one case, a massive cerebellar edema developed, which could be explained by the large sigmoid sinus thrombosis. What is more, all of the patients remain asymptomatic after long follow-up. Our results support the conclusions of other authors $[1,2,3,5,8]$ that postoperative DST is rarely symptomatic in such cases. This is likely due to collateral venous drainage, making thrombosis well-tolerated.

Risk factors

Duration of the surgery, the tumor volume, side, the patient's age or sex were not risk factors of postoperative thrombosis. Moreover, we did not observe the correlation between thrombosis and smaller sinus diameter observed by other authors $[1,3]$. It is an interesting fact, conflicting with the assumption that lower caliber of the vessel and slower flow could increase the risk for thrombosis in that group of patients. The most important risk factor seems to be an intraoperative injury of the sinus, which was observed in $23 \%$ of cases in the thrombosis group and only in $3 \%$ of cases in the non-thrombosed group. It means that sinus exposure is the main risk factor of thrombosis and the main prophylactic activities should focus on minimizing the sinus manipulation. Some authors theorized that even heat conduction during bone drilling, could facilitate the thrombotic cascade [1]. In our group, the most common moment of sinus injury was the moment of passing the footplate parallel to the sigmoid sinus. We have noticed that in most of such cases we injured not the sigmoid sinus itself, but the mastoid emissary vein which connects the venous drainage of the brain with the superficial scalp venous network. During craniotomy, the vein was usually ruptured and avulsed, causing also bleeding from the sigmoid sinus. We manage that complication by changing the strategy of craniotomy. Nowadays, we perform craniotomy to the point where the vein exits the skull through the foramen. The remaining part of the bone is drilled using the high-speed bone cut and diamond drills, to the level of the medial margin of the sinus, which is sufficient for the vestibular schwannoma surgery. After such modification of the craniotomy technique, we observed a markedly lower incidence of the postoperative sinus thrombosis. However, further analysis on that topic is needed.

Incidence

Previously, the incidence of DST after posterior fossa surgery was estimated at $11.6 \%$ [8]. Recent studies have noted more frequent diagnoses of thrombosis - 32.4\% [3], 38.9\% [9], however, there are studies with lower frequency - $6 \%$ [1], 6.7\% [10]. The reported incidence depends on the character of the study retrospective or prospective, the imaging modality - contrast enhanced or not and on the surgical approach. In our retrospective study, where every analyzed case was investigated using contrast enhanced MRI, we noted postoperative changes in $22 \%$. It must be noted that in 7 cases the sinus was narrowed by the hemostatic agent used for the dural closure, which does not mean thrombosis at all. 
True thrombosis was noted in 19 cases, which account for $16 \%$ of the studied patients. As mentioned above, the studies analyzed lesions of various surgical approaches, different pathologies, non-contrast postoperative examinations. To the best of our knowledge, our study is the largest study focusing on vestibular schwannoma operated on using the retrosigmoid approach with postoperative contrast enhanced MRI.

Classification

During the analysis of the postoperative imaging we found various presentations of the lateral sinuses after retrosigmoid craniotomy. In 7 cases we did not found any loss of contrast filling inside the sinus lumen, however, the flow was compromised by compression caused by the hemostatic agent. None of the patients from that group developed any symptoms. Long-term postoperative control revealed complete resolution of compression in 5 cases, partial resolution in 1 case. One patients was lost to follow-up. Such presentation seems to be benign and asymptomatic. To the best of our knowledge, such radiological symptom was never described nor analyzed in the neurosurgical literature.

In 19 cases, we managed to visualize true thrombosis of the sinus. In $31 \%$ of cases, the extent of the thrombus was compromising less than a half of the sinus diameter. In the rest of the cases, the thrombus size was larger. In that group, we managed to highlight the differences in the radiological pattern. In $23 \%$ of cases, thrombosis was filling more than a half of the sinus diameter. In $8 \%$, it filled almost all crosssection of the sinus, leaving marginal flow of the contrast around the thrombus. In another $8 \%$ of cases, we noted complete obstruction of the sinus, however, without engorgement of the veins draining into the sinus. Moreover, in $3 \%$ of all cases we found a complete thrombosis with such engorgement. To the best of our knowledge, there is no classification of postoperative dural sinus thrombosis in the literature. The authors of previous studies managed the topic noting only the fact of presence or absence of the thrombus.

Anticoagulation and management

The main concern in dural sinus thrombosis is prevention of the thrombus propagation, obstruction of the blood drainage and increased intracranial pressure due to hyperemia or intracerebral hemorrhage. Such a situation could be fatal, as reported [5]. The role of anticoagulation is to prevent such thrombus progression. However, the usage of anticoagulants in the early postoperative course is always a dilemma. What is more, there are no guidelines managing that topic. Despite the relative clinical safety of the lowmolecular-weight heparine (LMWH) in the prophylactic dosage, the use of higher, therapeutic doses seems to be more risky and thus is avoided postoperatively. However, even low dosage could cause hemorrhagic complications, as reported by other authors [1]. In our group, we did not observe any complications which could be associated with the used anticoagulation. We manage thrombosis basing on its extent. The sinus compression or a small thrombus was managed only by administering IV fluids and clinical observation. In more prominent cases, we administered LMWH in prophylactic dosage and fluids. Anticoagulation was administered in 10 cases (38\%), never earlier than 24-48 hours after the surgery, and was usually discontinued before the discharge. In all cases we used enoxaparin. Based on 
our results, it is debatable if anticoagulation is necessary in such cases. Almost all of them were asymptomatic. In some of the cases, the thrombus was noticed retrospectively and such patients had been managed without anticoagulation. Only one case, described earlier, could be associated with venous thrombosis. However, in that particular case, the superior petrosal vein was closed during the surgery, so the explanation of that case is complex. On the other hand, we did not observe any hemorrhagic complications in that group of patients. Using our thrombosis classification, we hypothesize that the cases where a thrombus is causing the engorgement of other draining veins (grade $\mathrm{VI}$ ) are the most important indication for the administration of anticoagulants in that group of patients. However, such a situation was observed only in 1 patient ( $4 \%$ of all thrombotic cases).

Limitations

In our study we have analyzed the largest group of the vestibular schwannomas operated on using the suboccipital retrosigmoid approach. What is more, it is the largest group examined with the postoperative MRI, which is superior in the diagnostics of thrombosis to routine non-contrast CT. We attempted to classify the postoperative changes in the lateral venous sinuses and develop a classification which could help further investigators. Additionally, we performed a clinical and radiological follow-up of most of the patients, giving the natural history of postoperative dural sinus thrombosis.

However, it is the experience of one institution only. Moreover, the presented study is retrospective and based only on contrast enhanced MRI (DSA was not performed). A large, multicenter prospective trial is required for the high-level guidelines for management of postoperative dural sinus thrombosis after vestibular schwannoma resection.

\section{Conclusions}

Postoperative changes in the dural venous sinuses are a frequent finding after vestibular schwannoma surgery using the suboccipital retrosigmoid approach. Intraoperative dural injury is a risk factor for thrombosis. Thrombosis in that group of patients is usually asymptomatic and does not influence the prognosis.

\section{Declarations}

\section{Statements and Declarations}

Funding - The authors did not receive support from any organization for the submitted work.

Conflicts of interest/Competing interests - The authors have no relevant financial or non-financial interests to disclose.

Availability of data and material - available

Code availability (software application or custom code) - not applicable

Ethics approval - The Institutional Ethics Committee was informed about the ongoing study, and the Committee stated no need for its approval. 
Consent to participate (include appropriate statements) - Informed consent was obtained from all individual participants included in the study.

Consent for publication - Not applicable. No data, which could lead to identification of the patient (photography, names) was used.

Authors' contributions:

Conceptualization: Kamil Krystkiewicz.

Methodology: Kamil Krystkiewicz.

Investigation: Kamil Krystkiewicz, Dawid Wrona, Marcin Tosik, Anna Morawska, Konrad Stopa, Cezary Wałęsa.

Formal analysis and investigation: Kamil Krystkiewicz, Marcin Birski, Łukasz Szylberg

Writing - original draft preparation: Kamil Krystkiewicz.

Writing - review and editing: Marcin Birski, Marcin Tosik, Marek Harat.

Resources: Kamil Krystkiewicz, Jacek Furtak, Marcin Birski, Marek Harat.

Supervision: Marek Harat.

\section{References}

1. Abou-Al-Shaar H, Gozal YM, Alzhrani G, Karsy M, Shelton C, Couldwell WT. (2018) Cerebral venous sinus thrombosis after vestibular schwannoma surgery: a call for evidence-based management guidelines. Neurosurg Focus. 45(1):E4.

doi: 10.3171/2018.4.FOCUS18112.

2. Apra C, Kotbi O, Turc G, et al. (2017) Presentation and management of lateral sinus thrombosis following posterior fossa surgery. J Neurosurg. 126(1):8-16. doi: 10.3171/2015.11.JNS151881

3. Benjamin CG, Sen RD, Golfinos JG, et al. (2018) Postoperative cerebral venous sinus thrombosis in the setting of surgery adjacent to the major dural venous sinuses. J Neurosurg. Oct 1-7. doi: 10.3171/2018.4.JNS18308.

4. Chibbaro S, Cebula H, Todeschi J, et al. (2018) Evolution of Prophylaxis Protocols for Venous Thromboembolism in Neurosurgery: Results from a Prospective Comparative Study on LowMolecular-Weight Heparin, Elastic Stockings, and Intermittent Pneumatic Compression Devices. World Neurosurg. 109:e510-e516. doi: 10.1016/j.wneu.2017.10.012

5. Guazzo E, Panizza B, Lomas A, et al. (2020) Cerebral Venous Sinus Thrombosis After Translabyrinthine Vestibular Schwannoma-A Prospective Study and Suggested Management Paradigm. Otol Neurotol. 41(2):e273-e279. doi: 10.1097/MA0.0000000000002508. 
6. Keiper GL Jr, Sherman JD, Tomsick TA, Tew JM Jr (1999). Dural sinus thrombosis and pseudotumor cerebri: unexpected complications of suboccipital craniotomy and translabyrinthine craniectomy. $J$ Neurosurg. 91(2):192-197. doi: 10.3171/jns.1999.91.2.0192.

7. Kow CY, Caldwell J, Mchugh F, Sillars H, Bok A. Dural venous sinus thrombosis after cerebellopontine angle surgery: Should it be treated?. J Clin Neurosci. 2020;75:157-162. doi:

10.1016/j.jocn.2019.12.036

8. Moore J, Thomas P, Cousins V, Rosenfeld JV. (2014) Diagnosis and Management of Dural Sinus Thrombosis following Resection of Cerebellopontine Angle Tumors. J Neurol Surg B Skull Base. 75(6):402-408. doi: 10.1055/s-0034-1376421

9. Sawarkar DP, Verma SK, Singh PK, Doddamani R, Kumar A, Sharma BS. Fatal Superior Sagittal Sinus and Torcular Thrombosis After Vestibular Schwannoma Surgery: Report of a Rare Complication and Review of the Literature. World Neurosurg. Dec;96:607.e19-607.e24. doi:

10.1016/j.wneu.2016.09.075.

10. Shew M, Kavookjian H, Dahlstrom K, et al. (2018) Incidence and Risk Factors for Sigmoid Venous Thrombosis Following CPA Tumor Resection. Otol Neurotol. 39(5):e376-e380. doi: 10.1097/MAO.0000000000001806.

\section{Figures}



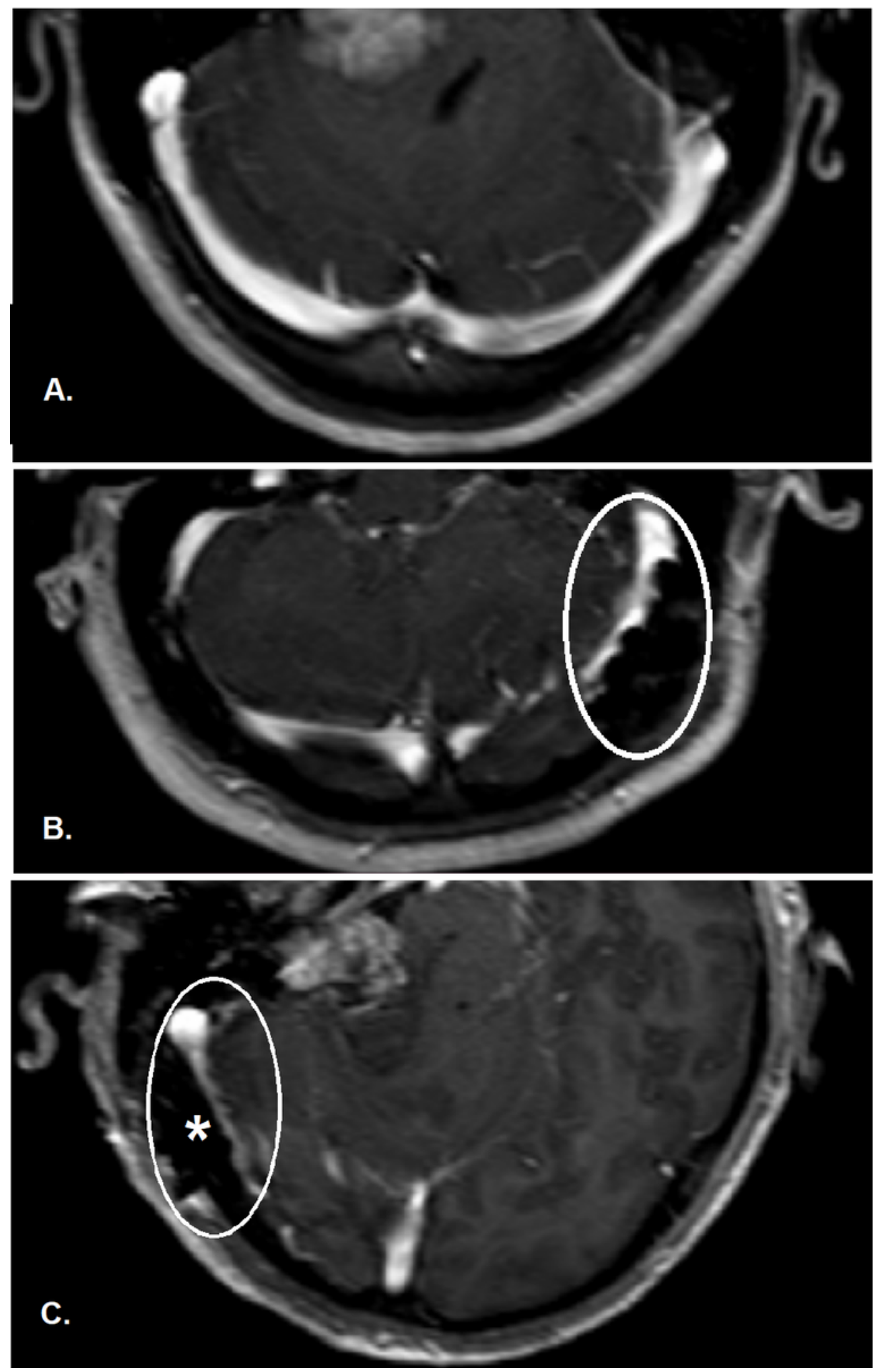

\section{Figure 1}

Grade 1 - external compression by the hemostatic agent. A. Normal, preoperative image of the noncompressed sinuses. B. Example of the external compression of the left sinus (encircled). Note the irregularities of the external border of the sinus, without thrombus within the lumen. C. Another example of the same grade - compressed right sinus 


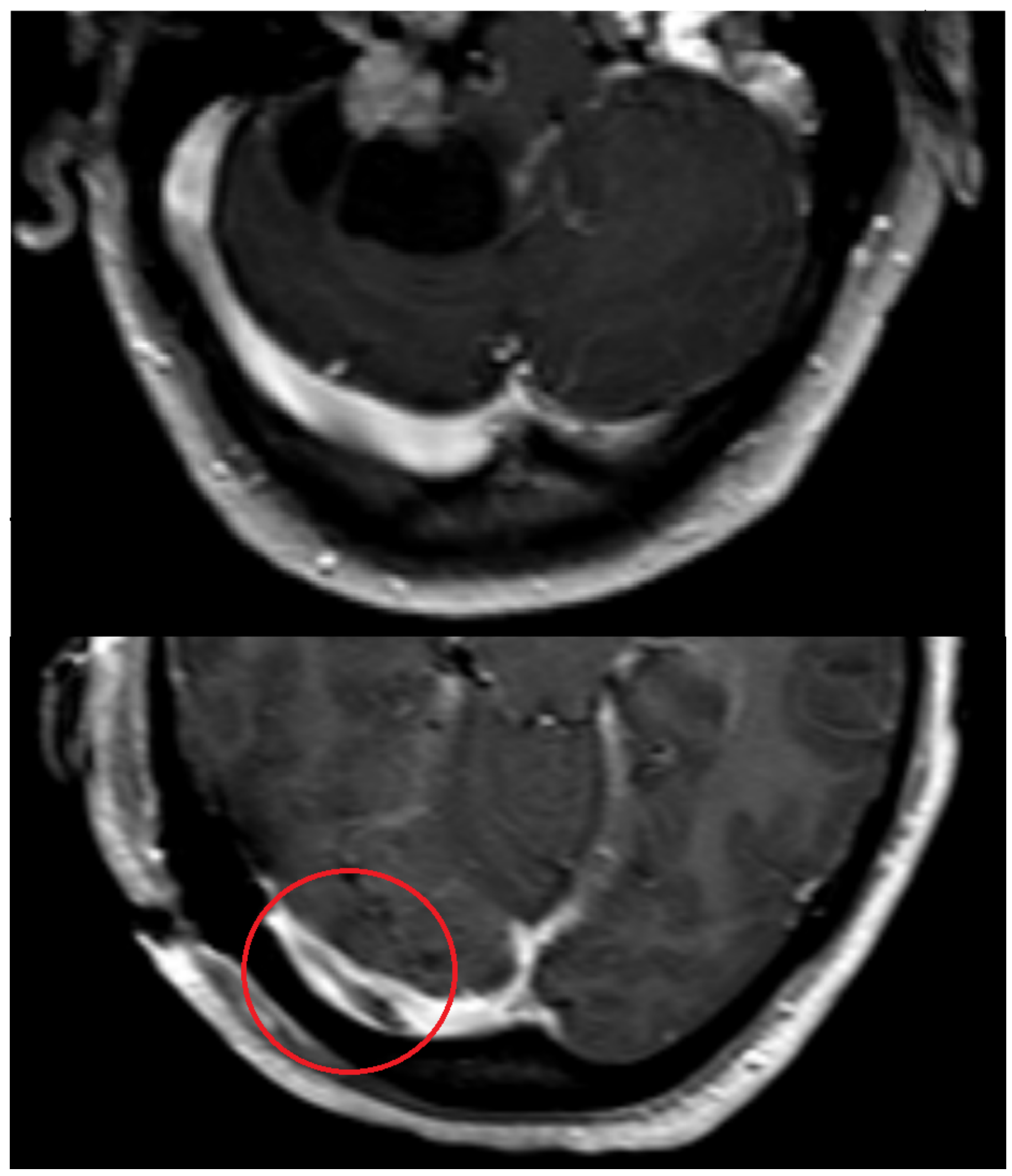

\section{Figure 2}

Grade 2 - Thrombus fills less than the $50 \%$ of the cross-section of sinus. Upper image - normal, preoperative MRI investigation. Lower image - large thrombus filling the transverse sinus on the right sight (encircled). Note that most of the sinus is patent 

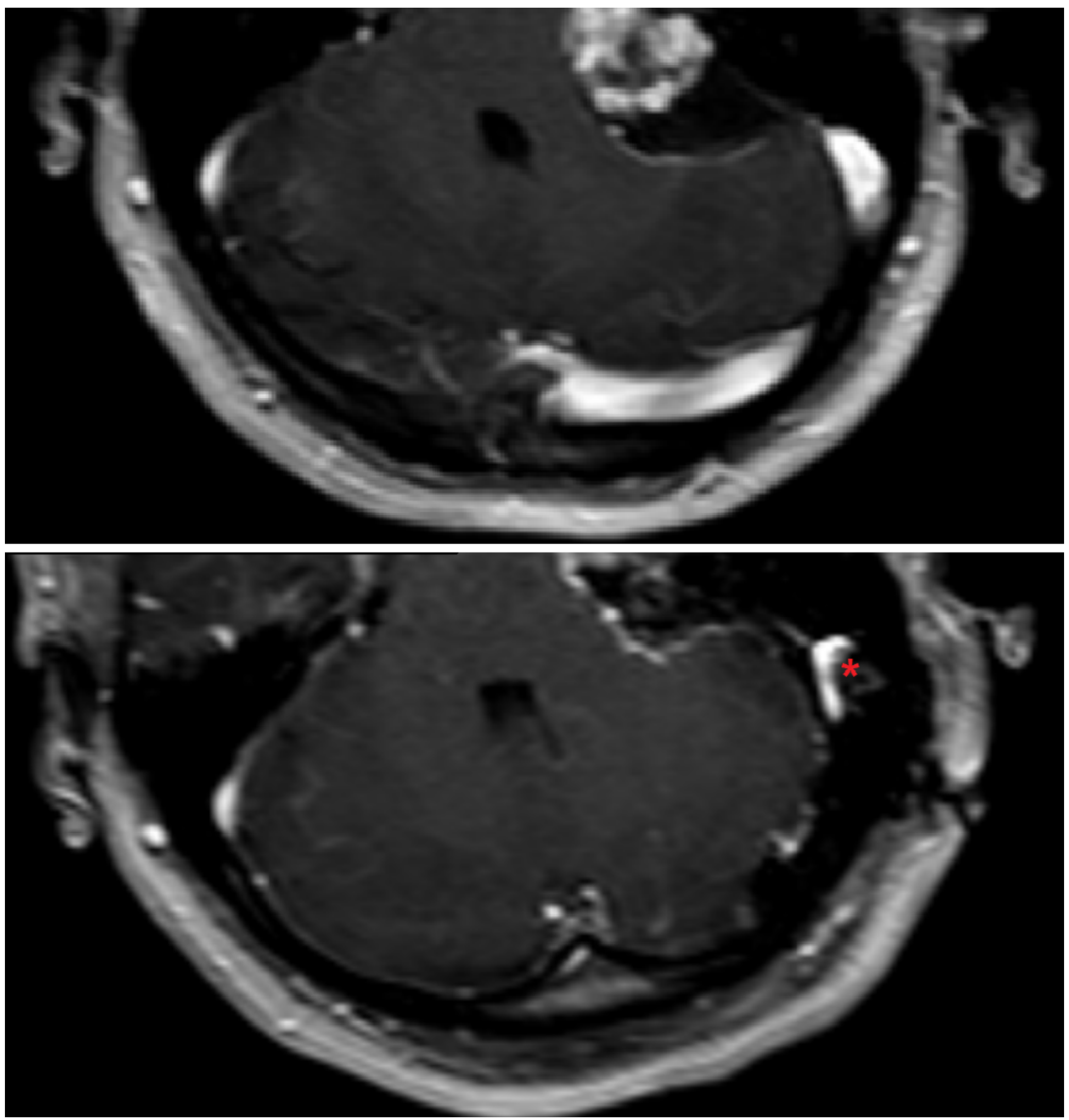

\section{Figure 3}

Grade 3 - Thrombus fills more than the $50 \%$ of the cross-section of sinus. Upper image - normal, preoperative MRI investigation. Lower image - postoperative MRI. * - intraluminal thrombus filling more than the $50 \%$ of the cross-section of sinus 


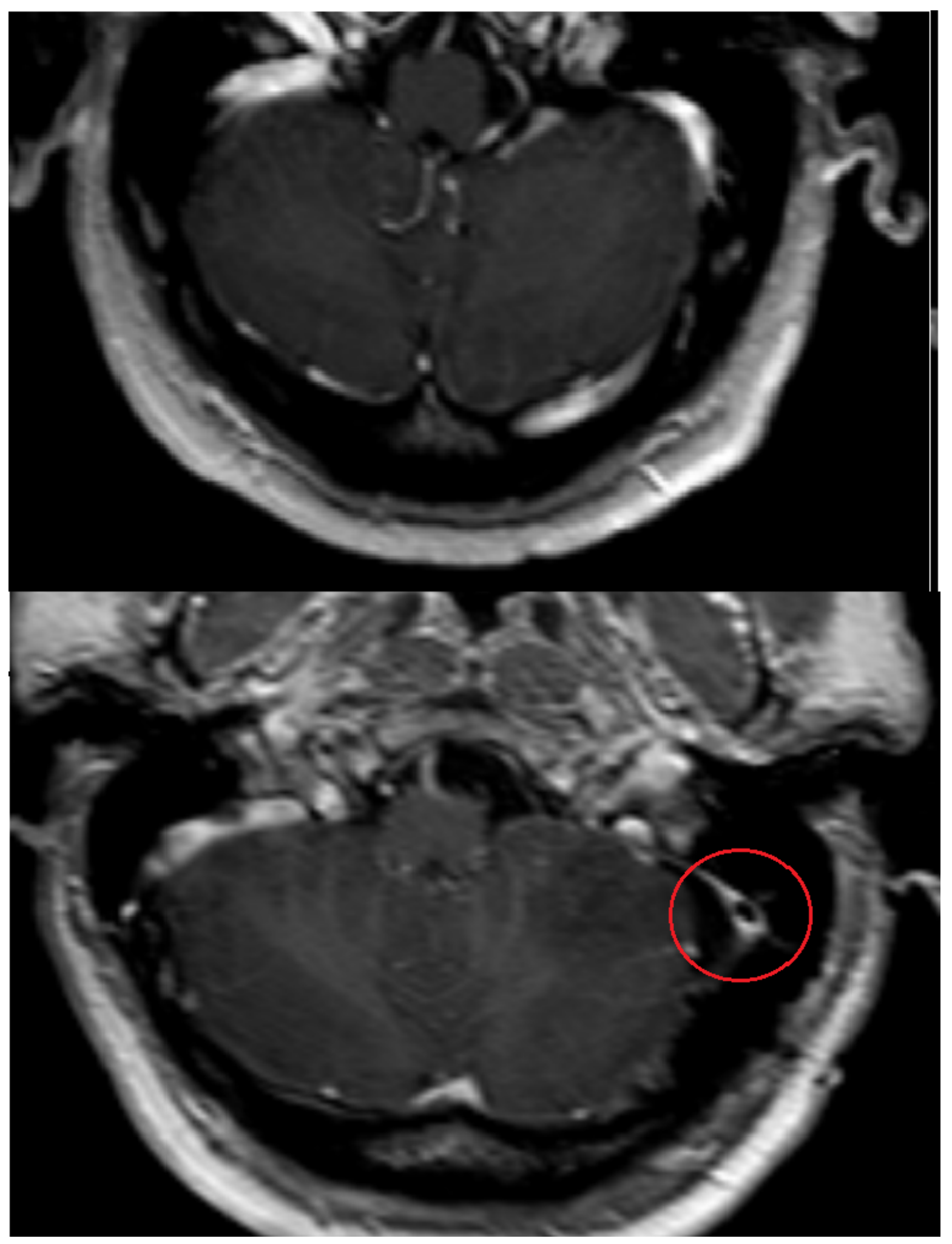

\section{Figure 4}

Grade 4 - Almost complete closure of the sinus with the marginal flow around the thrombus. Upper image - normal, preoperative MRI investigation. Lower image - postoperative MRI. A large thrombus filling almost completely the cross-section of sinus (encircled). Note the marginal flow of the contrast between the thrombus and the wall of the sinus endothelium 

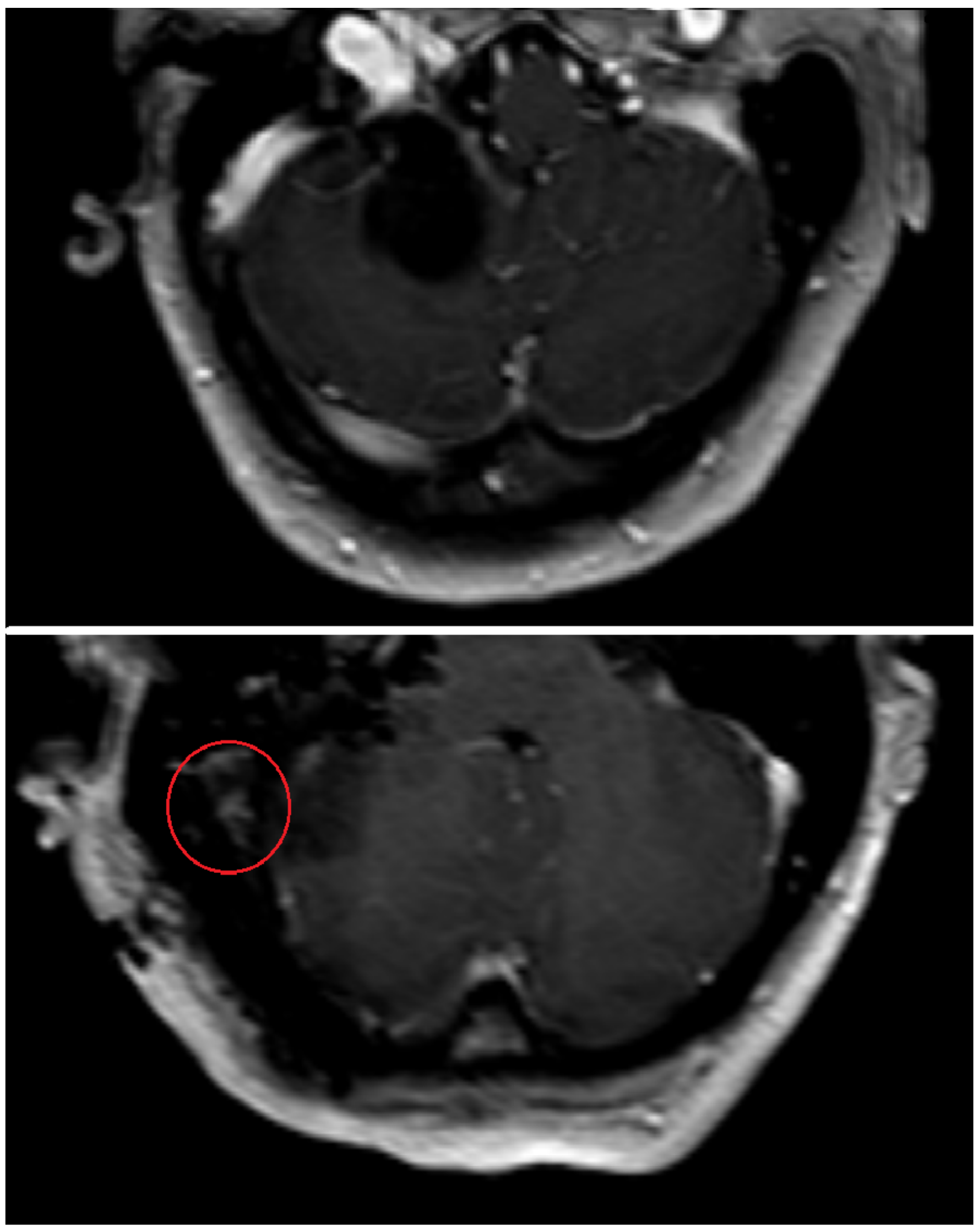

\section{Figure 5}

Grade 5 - Complete closure of the sinus without engorgement of the draining veins. Upper image normal, preoperative MRI investigation. Lower image - postoperative MRI. Complete closure of the right sigmoid sinus (encircled). Note that, there is no stasis in the veins draining into the sinus 

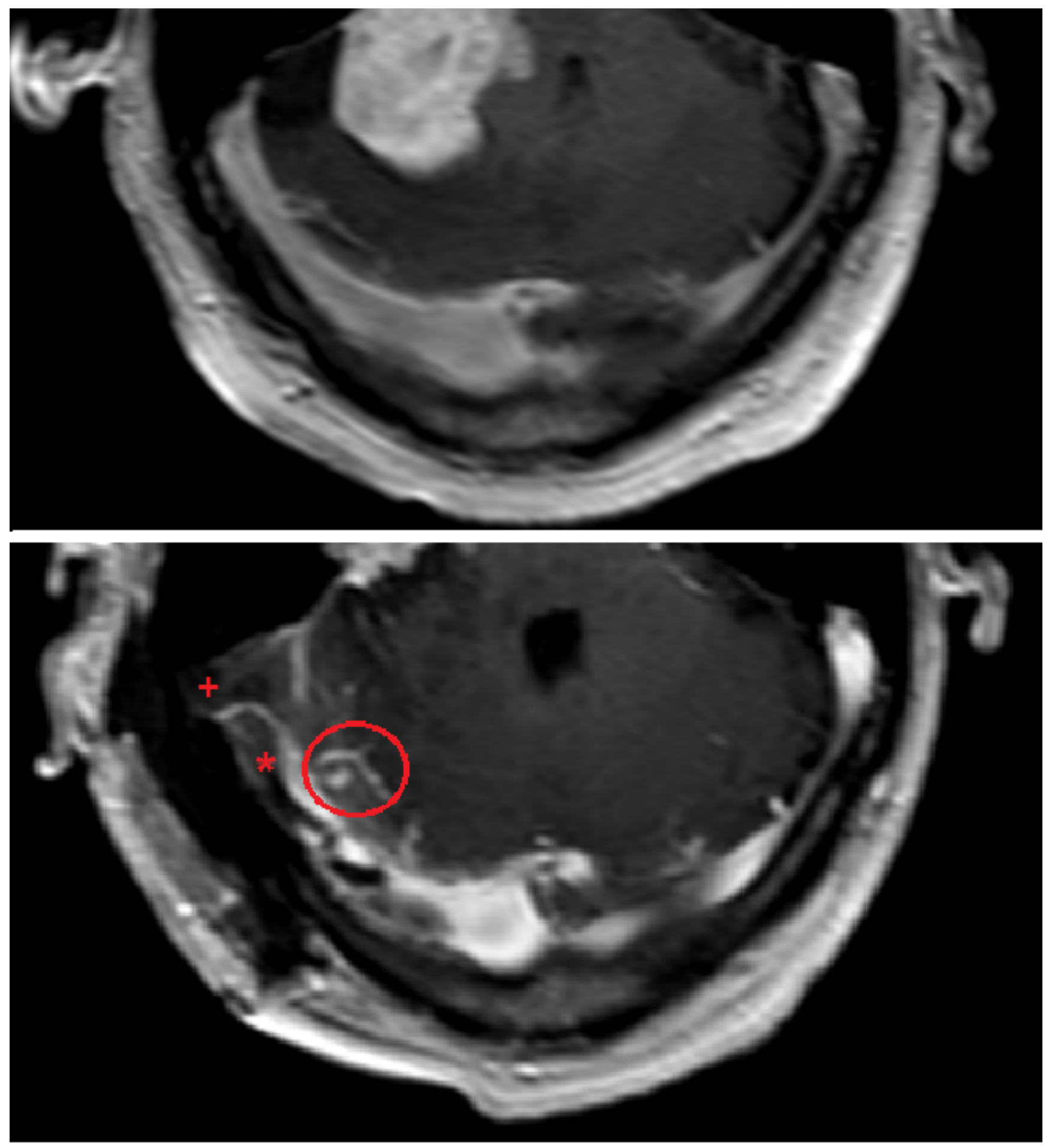

Figure 6

Grade 6 - Complete closure of the sinus with engorgement of draining veins. Upper image - normal, preoperative MRI investigation. Lower image - postoperative MRI. * - large thrombus filling the transverse sinus, occluding almost completely the lumen. + - complete obstruction of the sigmoid sinus. Note the enlarged draining, both infra- and supratentorial veins (encircled) 